Activity-based model for medium-sized cities considering external activity-travel: Enhancing FEATHERS framework

Peer-reviewed author version

FAZAL ABBAS BAQUERI, Syed; ADNAN, Muhammad; KOCHAN, Bruno \& BELLEMANS, Tom (2019) Activity-based model for medium-sized cities considering external activity-travel: Enhancing FEATHERS framework. In: Future generation computer systems, 96, p. 51-63.

DOI: 10.1016/j.future.2019.01.055

Handle: http://hdl.handle.net/1942/27862 


\title{
Activity-based Model for Medium-Sized Cities Considering External Activity-Travel: Enhancing FEATHERS Framework
}

\author{
Syed Fazal Abbas Baqueri ${ }^{\mathrm{a}, \mathrm{b}}$, Muhammad Adnan ${ }^{1, \mathrm{a}}$, Bruno Kochan $^{\mathrm{a}} \&$ Tom Bellemans ${ }^{\mathrm{a}}$ \\ ${ }^{a}$ UHasselt - Hasselt University, Transportation Research Institute (IMOB), Agoralaan, 3590 Diepenbeek, Belgium \\ ${ }^{\mathrm{b} C i v i l ~ E n g i n e e r i n g ~ D e p a r t m e n t, ~ D H A ~ S u f f a ~ U n i v e r s i t y, ~ K a r a c h i, ~ 75500, ~ P a k i s t a n ~}$
}

\begin{abstract}
Travel demand modeling has evolved from the traditional four-step models to tour-based models which eventually became the basis of the advanced Activity-Based Models (ABM). The added value of the ABM over others is its ability to test various policy scenarios by considering the complete activity-travel pattern of individuals living in the region. However, the majority of the ABM restricts residents' activities within the study area which results in distorted travel patterns. The external travel is modeled separately via external models which are insensitive to policy tests that an ABM is capable of analyzing. Consequently, to minimize external travel, transport planners tend to define a larger study area. This approach, however, requires huge resources which significantly deterred the worldwide penetration of ABM. To overcome these limitations, this study presents a framework to model residents' travel and activities outside the study area as part of the complete activity-travel schedule. This is realized by including the Catchment Area (CA), a region outside the study area, in the destination choice models. Within the destination choice models, a top-level model is introduced that specifies for each activity its destination inside or outside the study area. For activities to be performed inside the study area, the detailed land use information is utilized to determine the exact location. However, for activities in the CA, another series of models are presented that use land use information obtained from open-source platforms in order to minimize the data collection efforts. These modifications are implemented in FEATHERS, an ABM operational for Flanders, Belgium and the methodology is tested on three medium-sized regions within Flanders. The results indicate improvements in the model outputs by defining medium-sized regions as study areas as compared to defining a large study area. Furthermore, the Points of Interests (POI) density is also found to be significant in many cases. Lastly, a comprehensive validation framework is presented to compare the results of the $\mathrm{ABM}$ for the medium-sized regions against the ABM for Flanders. The validation includes the (dis)aggregate distribution of activities, trips, and tours in time, space and structure (e.g. transport modes used and types of activities performed) through eleven measures. The results demonstrate similar distributions between the two ABM (i.e. ABM for medium-sized regions and for Flanders) and thus confirms the validity of the proposed methodology. This study, therefore, shall lead to the development of ABM for medium-sized regions.
\end{abstract}

Keywords: Activity-based Model, External Activity-Travel, External trips, FEATHERS, Activity-based model validation.

\section{Introduction}

The notion that the need for activity participation derives its associated travel, led to the formation of the Activity Based Model (ABM) (Ben-akiva et al., 1996). A typical ABM considers the complete daily activity-travel pattern of individuals living in the study area. This includes, for each agent in the synthetic

\footnotetext{
${ }^{1}$ Corresponding Author

E-mail addresses: fazal.abbas@dsu.edu.pk (Syed Fazal Abbas Baqueri), muhammad.adnan@uhasselt.be (Muhammad Adnan), bruno.kochan@uhasselt.be (Bruno Kochan), tom.bellemans@ uhasselt.be (Tom Bellemans)
} 
population, the number of activities to be performed and specific attributes of each activity: type, start time, duration, and location. Furthermore, these simulated activities are also linked together via a travel component having its own dimensions: travel time, travel mode. Finally, the tours are formed. Therefore, the added benefits of an ABM over a four-step model are its unit of analysis from zones to individuals and the consistency between the submodels that ensures a consistent travel pattern. In reality, subjected to the attractiveness of the study area and its surrounding region, some of the activities can be performed outside the study area which results in residents Internal-External (IE) trips. However, the majority of the ABM does not model IE trips as they allow the destination choice of activities only within the modeling region, e.g. DAYSIM, ABM within SimMobility (Singapore) and FEATHERS (Flanders, Belgium, and Seoul, South Korea) (Adnan, Pereira, Miguel, et al., 2016; Bellemans et al., 2010; Bowman \& Bradley, 2006). The ABM output is fed in the route assignment along with internal-external trips obtained from other models. Such an approach may result in the following deficiencies:

- Overestimating trips and activities within the region by assigning all residents' activities within the study area while completely disregarding the residents' external activities and trips.

- A double representation of residents' external trips at the route assignment stage, i.e. 1) from the $\mathrm{ABM}$ where external activity-travel of individuals is considered as internal trips and 2) through the output from the external trips model.

- Inability to test policy applications on resident's external travel because these are estimated outside the scope of the ABM.

These limitations have been well recognized and to overcome them, modelers tend to define a more extensive study area. Although this practice may reduce overall external travel, it increases the data collection and model development efforts: collecting household travel survey (HTS) data for a larger study area, preparing its synthetic population and running the ABM.

Consider a case of East Hampshire District Council (EHDC) - a medium-size district in the South East Region of England approximately 100km away from London. Expectedly, a lot of individuals commute from EHDC to London. Therefore, a travel demand model for EHDC should also include East of England and London Regions (formally government office region) in the study area (as recommended in Department for Transport 2017, p.13). This expansion of the study area results in unwanted model complexities such as modeling the travel behavior of Londoners which is indeed not the central objective. Likewise, expanding the study area may not always be a solution because of for example a boundary between two countries, resulting in data collection issues. For instance, the present ABM for Singapore (Siyu, 2015) is subjected to this issue as it assigns the residents' activities within Singapore, whereas, a lot of individuals frequently travel to Malaysia. As a result, the resident/s trips are over assigned within Singapore while completely ignoring their external travel. Detailed practical examples of these limitations are defined in Baqueri et al. (2018).

Consequently, only a few ABMs are operational at present mainly subjected to huge data collection and resources. Whereas, in order to develop a travel demand model for a medium-sized region, modelers have to rely on conventional four-step models. Therefore, it can be safely stated that the ability to model residents' external travel within ABM shall pave the way to develop an ABM for a medium-sized region. In light of these concerns, this study presents a framework to model residents external trips in FEATHERS - an activity-based travel demand model (Bellemans et al., 2010). The framework includes 1) defining an external region as Catchment Area (CA) within the ABM and 2) inclusion of CA within destination choice set. To limit the data collection efforts, the land use information of the CA is solely obtained using the opensource information to minimize the data collection cost. The study also describes the application of the proposed framework in three medium-sized study areas in Flanders, Belgium. Furthermore, a validation 
framework for $\mathrm{ABM}$ along with its implementation is also presented to compare the results of the proposed model against the model without a $\mathrm{CA}$.

The rest of the paper is arranged as follows. The next section summarizes the literature on modeling external travel within ABM and ABM validation. The third section describes the modified FEATHERS framework. The fourth section describes the case study: the implementation study areas and the model results for each. The fifth section describes a framework for model validation along with aggregate and disaggregate validation. The sixth section provides a discussion of results and validation and the last section presents the 92 conclusion.

\section{Literature review}

\subsection{Activity-Based Model}

Since their inception, the activity-based models have achieved significant progress in terms of theory, implementation, and deployment. Researchers and practitioners, particularly in the USA, Europe, and Japan develop and implemented ABMs. CARLA (constraint-based), STARCHILD (Recker et al., 1986a; Recker et al., 1986b), SCHEDULER (Gärling et al., 1994), DAYSIM (Bowman \& Ben-Akiva, 1998), TRANSIMS (Smith et al., 1995), and ALBATROSS (Arentze \& Timmermans, 2004) are some early examples of the ABM (Siyu, 2015, p.14).

ADAPTS (Agent-based Dynamic Activity Planning and Travel Scheduling), TASHA (Travel/Activity Scheduler for Household Agents) and SimMobility are some advanced prototypes of the ABM. These ABMs have much more sophisticated model structure to deal with the complex transport system (Auld \& Mohammadian, 2012; Miller \& Roorda, 2003; Adnan, Pereira, Miguel, et al., 2016). For instance, unlike other ABM frameworks, ADAPTS have an activity planning step that incrementally plans and updates activities for each individual for each time interval. TASHA models, for each individual in a household, its vehicle allocation, ridesharing and joint activities/trips. SimMobility integrates long-term models such as vehicle ownership, land use pattern with daily schedule and within day rescheduling such as disruption strategies. It also includes mode and destination accessibility for each individual through logsums.

With the passage of time, the spectrum of ABM has been constantly expanding to more advanced issues such as the demand for electric vehicles charging stations (Usman et al., 2017), Disruption Management Strategies (Adnan, Pereira, Azevedo, et al., 2016), carpooling demand (Hussain et al., 2016) and integration of autonomous vehicles in ABM (Childress et al., 2015). Recently, ABM has also demonstrated its multidisciplinary potential such as linking transportation with air quality analysis (Shabanpour et al., 2016), traffic noise (Kaddoura et al., 2017), energy demand and power-peaks (Weiss et al., 2017; Knapen et al., 2012), emissions and environmental impacts (Shiftan et al., 2015), and health assessments (Lefebvre et al., 2013). Therefore, it can be well guessed that the ABM will continue to maintain their impetus in future as well.

At present, most of the ABM disregard external travel and estimate them unconnectedly through other external models. The external trip models are analogues to first two steps of the four-step model as they predict aggregate external trip generation at external stations, i.e., highway intersections at the boundary of the study area and distribute them in the Traffic Analysis Zones (TAZs) of the study area. The travel mode for external trips is not explicitly modeled as usually cars are considered as travel mode and the OD matrix is directly used for route assignment along with the results of the ABM. Such an approach results in numerous problems as described in the previous section. However, few ABMs do consider the outside area through the additional zone(s) in the destination choice model. For example, ALBATROSS considers the surrounding area as one additional zone (Arentze \& Timmermans, 2004). Similarly, ADAPTS - a state-ofthe-art ABM, assigns external destinations to several zones around the Chicago region (Auld \& 

within the study area. Due to this, travel times and cost of trips between the study area and the surrounding region will be inappropriate and, therefore, sub-models within ABM that requires these inputs may not perform well. To address these stated concerns, this paper presents a comprehensive framework that includes the residents' external travel within the ABM framework.

\section{$134 \quad 2.2$ Activity-Based Model Validation}

135 Model validation is an important aspect. However, there are limited studies that describe validation of travel demand models (de Jong et al., 2007; Rasouli \& Timmermans, 2012). The studies vary according to the type of the model (rule based, utility based), aggregation level and uncertainty analyzed. Many studies described ABM validation by focusing on the discrete choice models, (Castiglione et al., 2003; Gibb \& Bowman, 2007; Bekhor et al., 2014) or a rule based approach (Zhuge et al., 2017; Cools et al., 2011; Bao et al., 2015; Bao et al., 2016; Rasouli, 2016). Majority of the studies focus on the core activity-scheduling part (Castiglione et al., 2003; Rasouli, 2016; Copperman et al., 2016). Most studies presented aggregate validation for different model kinds. For example, Bao et al. (2016) focused on two DTs only. Similarly, Copperman et al. (2016) described rail ridership. Bekhor et al. (2014) compared total vehicles kilometers travelled (VKT).

There is also a study that only described a generic validation framework for ABMs (Prelipcean et al., 2015). Drchal et al. (2016) described a Validation Framework for Activity-based Models (VALFRAM). The authors compared two basic system properties i.e. activities and trips across time, space and the structure (i.e. activity count and the travel mode used across activities). The study validated the model results using real-world activity-travel diary data and found a close relationship between both. Petrik et al.,(2018) discussed a variety of measures to compare the results of the two different model runs of an ABM in different settings to analyze model outcome uncertainty. They compare counts of tours, trips and stops for each activity, mode, location and a combination of them. The validation studies also vary with respect to the level of aggregation. For instance, Veldhuisen et al. (2000) compared origin-destination matrices at regional level. Furthermore, few studies also included socioeconomic attributes and described stratified model validation per population segment. Cools (2011) measured distance traveled across age and gender groups. Rasouli (2016) measured and presented validation results according to gender at the level of TAZs and study area. Besides these, Castiglione (2003) also included vehicle ownership in the validation criteria.

Literature suggests that the variation increases as the level of disaggregation increases. Therefore, it is important to assess model validation against individual attributes such as age, gender, vehicle ownership etc. Furthermore, rather than simply comparing the count, the emphasis should be on the distributions of activities and trips in time and space. Another important aspect for ABM validation is data availability. Since, an ABM not only needs to be validated for trips but also for activities, therefore, only traffic count data shall not suffice.

164 The above discussion emphasizes that it is essential to check the consistency of the model outputs when an knowledge, there exists no study that integrates residents' external trips within the ABM and presents its validation. This study aims to address these gaps. The validation measures proposed in this study can also be used for validating other extensions in the ABM.

\section{Research Framework}

170 This section describes a framework to model residents' external travel as part of the complete activity-travel 171 schedule in FEATHERS which is operational for Flanders, Belgium. A detailed functioning of FEATHERS 
172 is described in Bellemans et al. (2010), therefore, this paper only focuses on the components that are 173 developed or modified to include the resident external travel within the current framework (Figure 1). These 174 modifications include defining a CA, modifying destination choice models and the use of the open-source 175 land use data in the destination choice models. Within the activity pattern model, first, the number of work 176 episodes are determined followed by the generation of home-based tours. Then, for each tour, intermediate 177 activities are determined along with their placement i.e. before or after the tour's primary activity. The 178 intermediate activities are categorized as fixed [bring get, other] or flexible [shopping, services, social, 179 leisure and touring]. Once each of the activity in the schedule is determined then their duration is modeled. 180 The duration is categorized into three categories: short, medium and long. These categories have different 181 time ranges as per the activity type. For example, a medium shopping activity may have lesser duration than 182 a short leisure activity. For location choice, the first decision is the activity destination inside or outside the 183 study area. Based on this decision, relevant Decision Trees (DTs) are triggered to estimate accurate location 184 at the subzone level. The last step before the mode choice is the activity start time hour. At this moment 185 only the hour is determined when the activities will take place, exact timings are randomly chosen within 186 the 1-hour periods and are only available once all of the decisions have been made. The last decision is 187 related to the transport mode for each activity. For each following DT, the schedule decisions simulated 188 earlier are also included in the explanatory variables. The pseudo code of FEATHERS framework is shown 189 in Figure 2. 


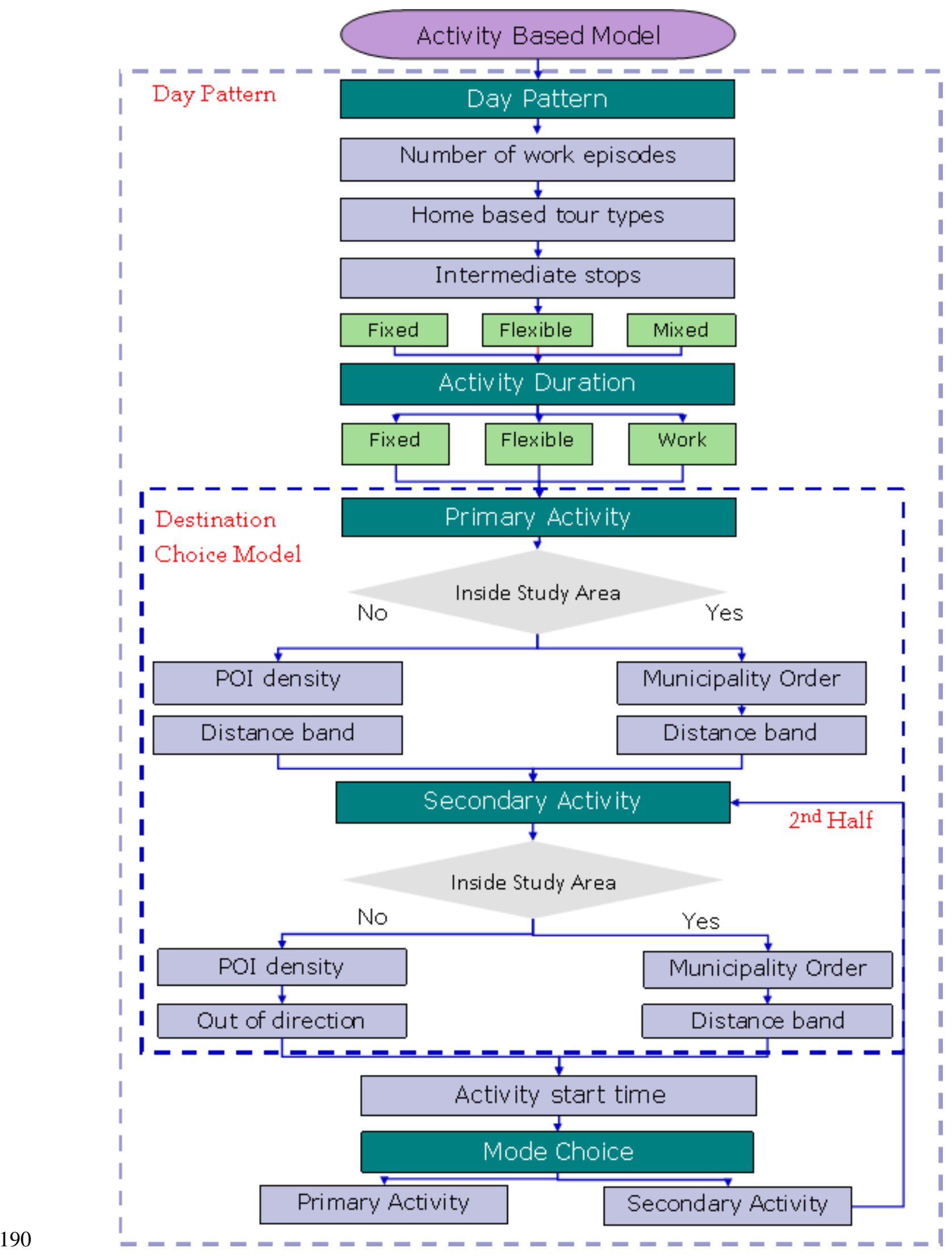

191 Figure 1: Framework to incorporate External activity-travel in Activity-Based Model FEATHERS 


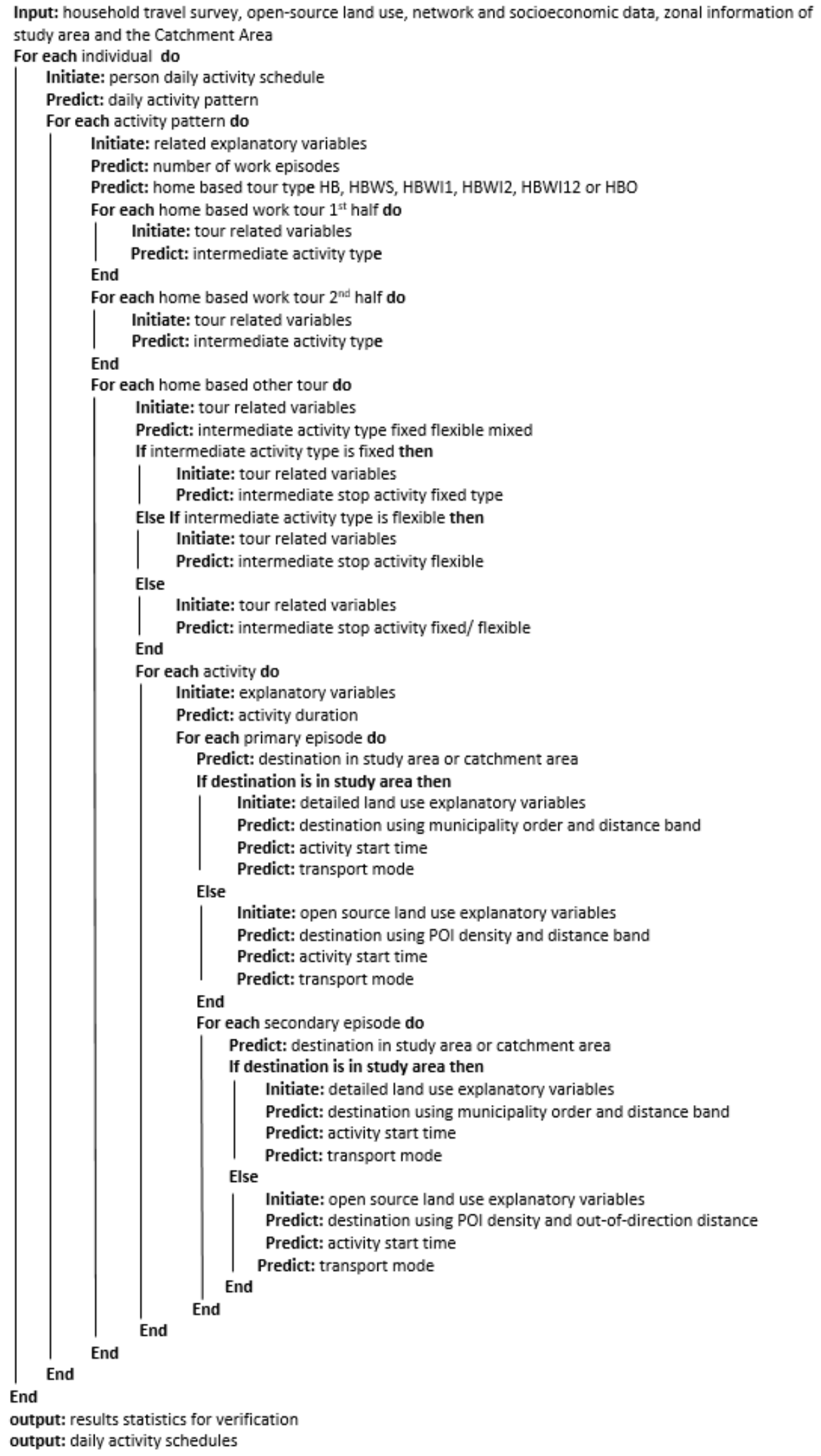




\subsection{Defining Catchment Area}

The primary region of interest for which an $A B M$ is to be developed is defined as the study area. The external region adjacent to the study area is defined as the CA. The spatial unit of the CA should be the same as of the study area to avoid inconsistencies in the models. The spatial units are defined in FEATHERS at three levels: superzones (municipalities), zones (city) and subzones (TAZs). Depending on the size, a municipality may contain more than one city and a city may contain more than one TAZs.

In the proposed approach, the first step is to define the study area as per the modeling needs and collect the HTS data from a sample population within the boundary of the study area. Then, based on the travel pattern of the individuals in the HTS, a CA is defined. The CA should be demarcated around the study area in a way such that it includes the farthest location that is used to perform an activity.

This goes without saying that few outliers such as exceptionally long-distance trips should be excluded before defining the CA. This exemption is observed because of various reasons. First, the number of trips decreases as the distance from the study area increases which makes the model development cumbersome with the limited observations. Second, the probability that the individuals performing such trips will return back to their home within the simulated time period (typically 24 hours) is very less. Therefore these trips should be modeled as long-distance trips through the framework defined by Baqueri et al. (2018). Third, in case of an international border in the CA, there are also other issues such as the inaccessibility to TAZs specifications and dissimilarity in land use data which may generate unwanted model complexities.

For example, consider developing an ABM for Mechelen; a city in Flanders (Dutch speaking part of Belgium) with Brussels and Antwerp in its vicinity. Based on the OVG - household travel survey data of Flanders (Janssens et al., 2014), around 30\% of the individuals travel outside Mechelen while the majority of the activities are performed within Flanders. Furthermore, only $1.4 \%$ of individuals commute to Wallonia (French-speaking part of Belgium) from Flanders due to the language barrier (Horckmans, 2017), which is quite low to train and test the model. Therefore, an ABM for Mechelen Flanders in included in the CA while Wallonia is discarded.

\subsection{Destination choice model}

The destination choice models in FEATHERS are built using DT with a multi-level decision hierarchy to specify the location of an activity. The first DT shortlists locations on the basis of predicted Municipality Order class. The municipality order is defined on the basis of attractiveness of a location and its distance from individual's current location. It is currently categorized in four categories, however, it can also be taken into continuous form when required. The second DT further narrow down locations on the basis of Distance Band (DB). The DB categorizes locations into classes on the basis of circular distance from the current location of the individual. Finally a location is randomly chosen from the remaining shortlisted locations belonging to the specified class of municipality order and the DB.

This methodology is first applied to the primary activity i.e. the main activity of the tour and then applied to the secondary activities of the tour. However, all decisions related to the primary activity are made first and then incorporated into the DTs of the secondary activities as the primary activity decisions directly influence on secondary activities.

\subsubsection{Top level models}

It is imaginable that the detailed land-use information, which has been obtained for the study area, may not be available for the CA. This is largely subjected to the limited resources or even unavailability of the information such as in case the study area is defined at the country level. Therefore, two top-level models are introduced in the current framework (shown in the decision box in Figure 1) each for the primary and 
the secondary activities which intent to identify if the activity will take place in the SA or the CA. If the activity will take place in the SA then the detailed information is used, otherwise, only the variables formulated from open source platforms are used in estimating sub-models. Land use characteristics such as type, opening time, area, and employment and transport network attributes such as travel time, transit availability, price, and frequency can be obtained from open source platforms for developing destination choice models, mode choice models and time-of-day models. Some examples of the relevant Open source platforms are OpenStreetMap (OSM) (OpenStreetMap contributors, 2017) and Google API (Google Developers, 2017)). This is the first decision for assigning locations to activities, therefore, it is referred as the top-level model.

Some may argue that the inclusion of the top-level models (to define if the activity shall be conducted in the $S A$ or the $C A$ ) in the decision hierarchy process is against the intuition as the SA boundary is simply a modeling term. While, in reality, an individual may not even be aware of the study area boundary let alone its inclusion in the decision process. However, this claim may not be true as the boundary of the study area has a practical significance whether it represents an international, provincial or a state-wide border or even a city-jurisdiction because individuals $d o$ consider these boundaries before choosing a destination.

For example, a Dutch citizen considers crossing the boundary between Netherlands-Belgium and Netherlands-Germany to commute as an equivalent to traveling 35 and 46 extra minutes respectively (Pieters et al., 2012). This border-crossing resistance is, however, less for shopping activity because of the same currency across the border. Similarly, the top-level model may also be relevant in case of inter-regional travel. For example, as mentioned above, on average only $1.4 \%$ of individuals commute to Wallonia from Flanders due to the language barrier (Horckmans, 2017). Likewise, the statewide travel demand models are widespread in the USA which validates the fact that the inter-state travel is not so common. Furthermore, this decision-making impression may also be valid for the ABMs that are developed at the metropolitanlevel and the boundary holds a toll cordon e.g. as in Paris during weekdays.

\subsubsection{New Decision Trees} The inclusion of a top-level model also affects other subsequent location choice decisions. Therefore, 15 DTs are developed/modified to accommodate for the modified decision-hierarchy process for destination choice.

Tour's main Activity is defined as primary activities in FEATHERS. The DT Choose Primary Location in Study Area or Catchment Area defines if the primary activity will be performed in the CA or not. The need for this DT is described in section 3.3.1. Depending on the location two more DTs are used to determine precise activity location, i.e. the TAZ where the activity shall be performed. For activities to be conducted inside the CA, the first DT is Choose POI Density Catchment Area that identifies the POI density class in which the activity shall be conducted. The second DT for determining location is Choose Distance Band Catchment Area that identifies the distance band in which the activity shall take place. The distance band and POI density here are discretized into five classes which can be modified as required. For activities that are to be taken place inside the study area, the same DTs are used as in the model without the CA.

Activities other than the tour's main activity are defined as secondary activities in FEATHERS. These are distinguished in the activity-skeleton according to their placement before or after the primary activity. The activities performed before the primary activity are considered as $1^{\text {st }}$ half while others are considered as $2^{\text {nd }}$ half. The DT Choose Secondary Location In Study Area Or Catchment Area $1^{\text {st }}$ half determines if the secondary activity that is to be conducted before the primary activity within the same tour will take place in or outside the study area. This is the top-level model for secondary activities (defined in section 3.2.1). For the activities to be taken place inside CA, the DT Choose Secondary Location in Catchment Area $1^{\text {st }}$ half is 
282 activated. An important variable in the DT is the out-of-direction travel distance which indicates that extent 283 to which an individual deviates from a straight line between home and the primary activity location 284 (equation 1). Similar DTs are used for determining locations of secondary activities that are to be performed 285 after the primary activities.

286 Out - of - direction distance $=\left[\right.$ distance $_{H}$ to $S L+$ distance $_{S L}$ to PL $-\left[\right.$ distance $_{H}$ to PL $]$ (1)

287 Where $\mathrm{H}=$ home location, $\mathrm{SL}=$ secondary location and $\mathrm{PL}=$ primary location

288 The DTs for CA solely rely on individual's socioeconomic attributes, land use information obtained from 289 open-source platforms, and already simulated activity-travel decisions from the higher order models but 290 they do not incorporate any detailed land use and network information as it may not be available for the CA.

\section{$291 \quad 3.4$ Relationship between open source and detailed land use information}

292 Since the open-source land use information is incorporated in the DTs, therefore, it is important to verify its 293 quality. This can be checked by comparing the open source land use information with the detailed land use 294 information available for the study area. Figure 3 compares the land use information of Flanders, Belgium 295 obtained from the official data source (Statbel, 2017) with the data obtained from the OpenStreetMap. The 296 results show a strong association between commercial land use area from the official data source and the 297 Points of Interest (POI) data from OpenStreetMap (OSM) in each Traffic Analysis Zone (TAZ). 298 Furthermore, besides commercial land use, few other land use types also have a strong correlation with the 299 POIs such as buildup and the transport land area ( 
300 Table 1). This association (between official and open source land use data) may differ from region to region, 301 but we believe a similar level of consistency of open source data, so our modeling methodology can be 302 valid.

303

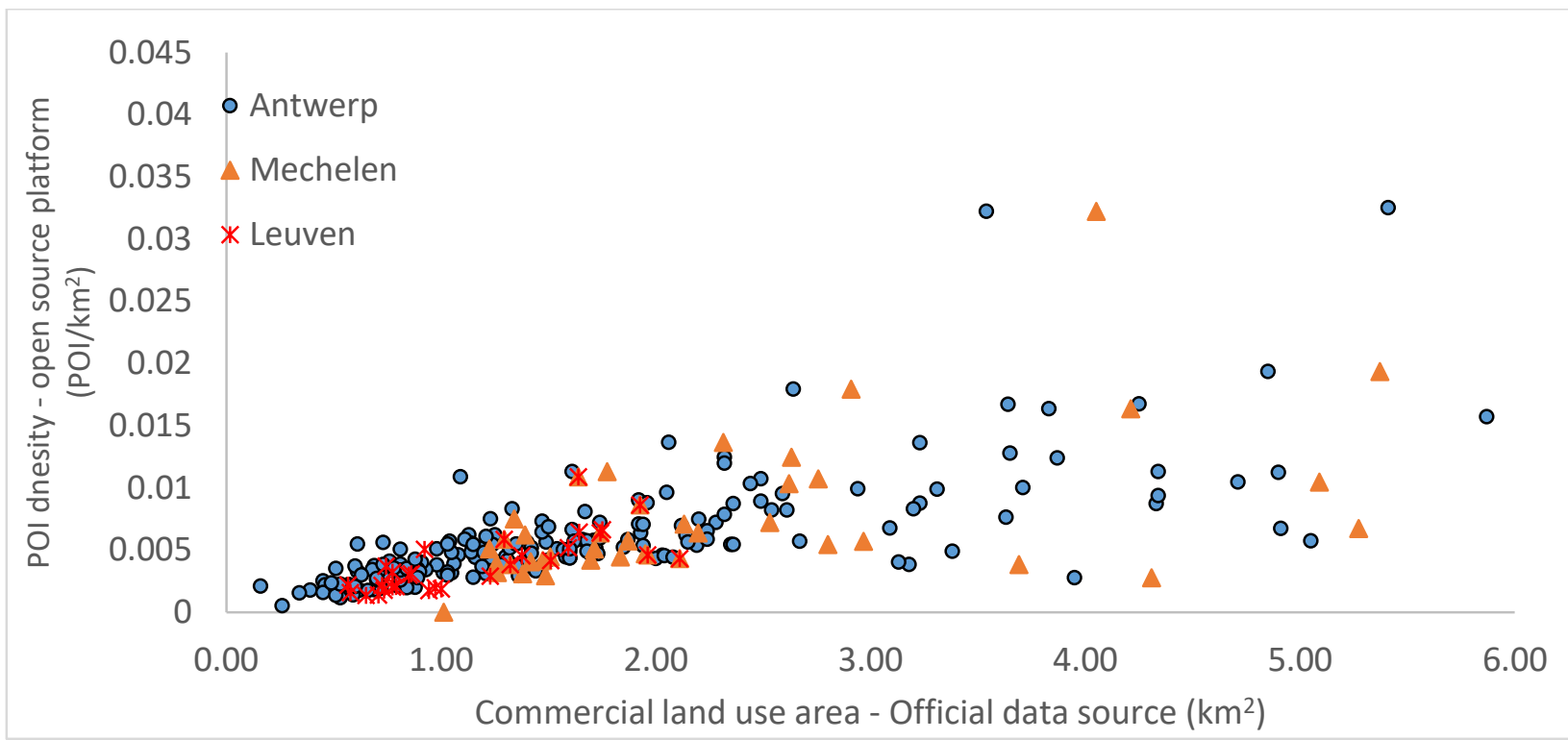




\begin{tabular}{|l|c|}
\hline Land use type $\left(\mathrm{km}^{2}\right)$ & Correlation with POIs (number) \\
\hline Commercial & $\mathbf{0 . 8 4}$ \\
\hline Buildup land & $\mathbf{0 . 5 4}$ \\
\hline Transport land & $\mathbf{0 . 5 1}$ \\
\hline Public & 0.47 \\
\hline Residential & 0.40 \\
\hline Recreation Open area & 0.34 \\
\hline
\end{tabular}

Highly correlated variables are marked in bold

\section{4. Case study}

This section describes the application of the above proposed FEATHERS framework on three study areas and the results obtained.

\subsection{Implementation study areas} following properties:

- Are medium-sized regions with a population between 0.5 to 1 million and an area around $1,000 \mathrm{~km}^{2}$

- Population density varies between 400 persons $/ \mathrm{km}^{2}$ to 1,000 persons $/ \mathrm{km}^{2}$.

- Around $25-35 \%$ of the residents perform external travel (obtained from BELDAM data (Hollaert et al., 2012)).

- Are a major trip attractor themselves and/or surrounded with a major trip attractor in their vicinity that influence external travel.

The details and the significance of these regions to test the proposed methodology are further defined.

\subsubsection{Antwerp region}

Antwerp region is located in the north of Flanders. It is the most populated province in Belgium with a population of 1.8 million. It is an attractive region with a port that generates a lot of commercial activity. Approximately $30 \%$ of the individuals tend to perform their activities outside the region, therefore, it shall be useful to check the distribution of activity types, and in particular work activities, in and outside the region.

\subsubsection{Mechelen region}

Mechelen is a home city for a lot of individuals who work in Brussels. Besides, Mechelen is equally distant between Brussels and Antwerp which makes it an interesting case to evaluate the proposed methodology. In order to define a relevant study area, a $20 \mathrm{~km}$ radius around Mechelen city is considered having a population of around 0.5 million. Approximately $34 \%$ of the residents perform external travel.

\subsubsection{Leuven region}

Leuven is located in Southern part of Flanders. It is surrounded by Brussels in its East which is an attractive region and attracts a lot of external travel. Therefore, it shall be interesting to implement this framework in Leuven region. The population of Leuven region is approximately 0.5 million and nearly $30 \%$ of the residents perform external travel. 


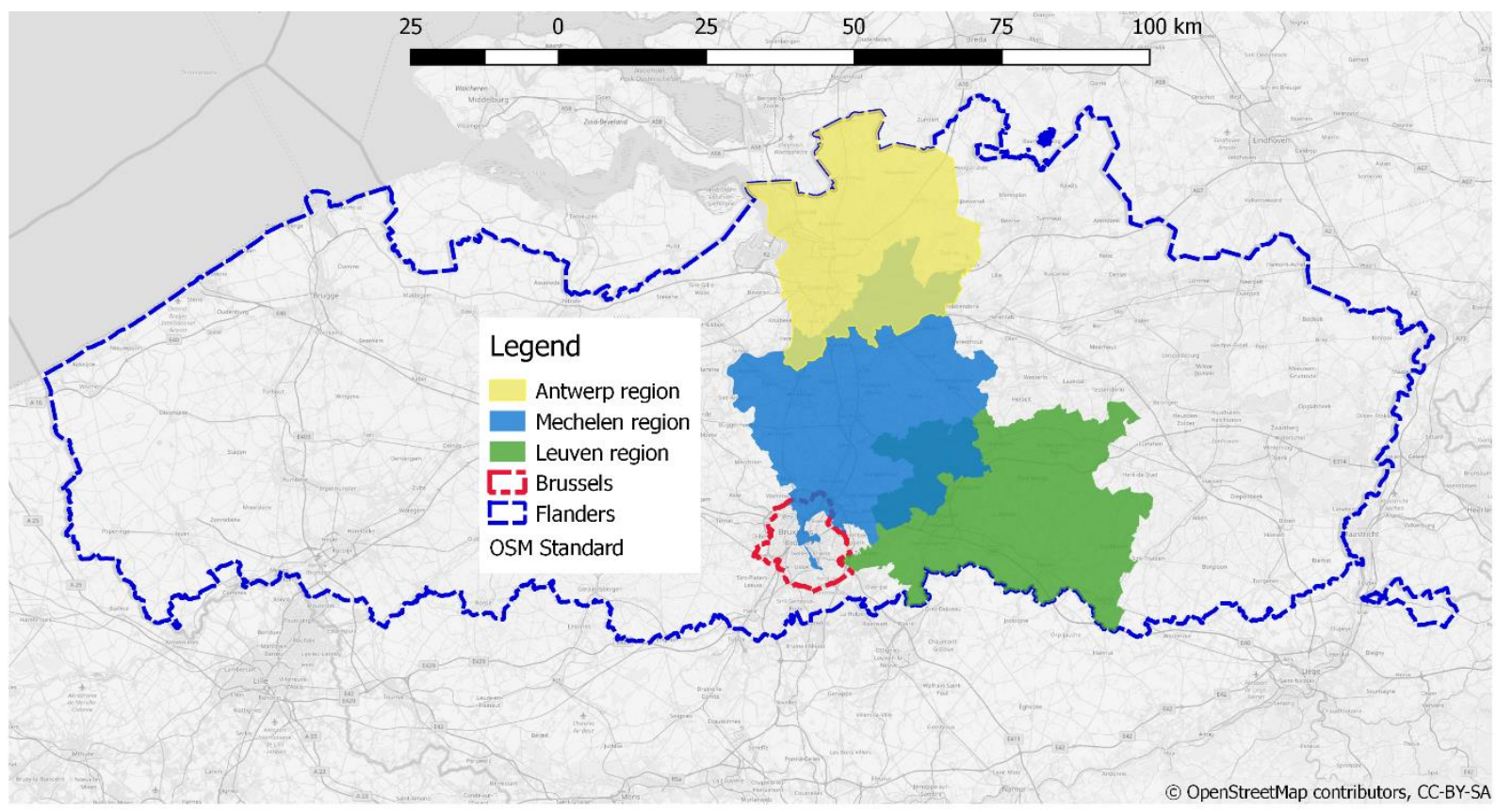

Figure 4: Study Areas Boundaries of Antwerp, Mechelen and Leuven region.

\subsection{Results}

The results obtained from running FEATHERS on these regions are described in this subsection. Only the individuals belonging to these study areas are used for model training and developing the synthetic population.

Table 2 compares the actual and predicted trips, tours, mode split and distribution of activities in the study area and Catchment Area. On aggregate, a close relationship is found between FEATHERS for full Flanders and for smaller study areas. For instance, earlier $23 \%$ of individuals performed an activity outside Antwerp region and in FEATHERS with CA setup 24\% performed at least one activity in CA. Similar results are obtained for Mechelen $(34 \% ; 31 \%)$. However, some differences are present in Leuven $(29 \% ; 36 \%)$. However, there are some differences in mode split in Antwerp region where a larger share of trips have been assigned to cars against public transport users.

Table 2: Aggregate results with and without Catchment Areas

\begin{tabular}{|l|l|l|l|l|l|l|}
\hline Parameter & \multicolumn{2}{|c|}{ Antwerp } & \multicolumn{2}{c|}{ Leuven } & \multicolumn{2}{c|}{ Mechelen } \\
\hline Peak Activity Start Time & $\begin{array}{l}\text { Without } \\
\text { CA }\end{array}$ & $\begin{array}{l}\text { With } \\
\text { CA }\end{array}$ & $\begin{array}{l}\text { Without } \\
\text { CA }\end{array}$ & $\begin{array}{l}\text { With } \\
\text { CA }\end{array}$ & $\begin{array}{l}\text { Without } \\
\text { CA }\end{array}$ & $\begin{array}{l}\text { With } \\
\text { CA }\end{array}$ \\
\hline Average Time spent travelling (min) & 44.31 & 46.74 & 57.48 & 60.79 & 55.65 & 52.43 \\
\hline \% of trips in Peak hour & 8.59 & 9.93 & 8.90 & 9.34 & 8.9 & 8.5 \\
\hline Work Activity \% & 23.54 & 23.02 & 26.54 & 24.91 & 26.54 & 25.42 \\
\hline Education Activity \% & 15.99 & 13.47 & 21.91 & 15.91 & 21.92 & 12.94 \\
\hline Daily Shopping Activity \% & 21.51 & 20.60 & 15.28 & 21.99 & 15.28 & 19.35 \\
\hline Non-Daily Shopping Activity \% & 8.28 & 10.10 & 7.46 & 8.77 & 7.46 & 8.27 \\
\hline Services Activity \% & 10.15 & 12.06 & 9.12 & 8.65 & 9.12 & 10.81 \\
\hline Car\% & 42.23 & 46.8 & 48.93 & 47.52 & 48.94 & 46.49 \\
\hline
\end{tabular}




\begin{tabular}{|l|l|l|l|l|l|l|}
\hline Public Transport \% & 10.36 & 9.16 & 29.68 & 10.64 & 7.6 & 11.84 \\
\hline Non-Motorized transport \% & 31.07 & 24.17 & 7.59 & 23.23 & 29.68 & 24.06 \\
\hline Simple tour \% & 62.35 & 63.68 & 60.58 & 60.60 & 64.34 & 20.40 \\
\hline 2-activity tours \% & 21.73 & 21.26 & 22.61 & 22.49 & 20.40 & 9.24 \\
\hline \% of work Activities in CA & 33.96 & 30.61 & 45.08 & 44.6 & 35.85 & 38.06 \\
\hline \% on individuals travelling to CA & 22.6 & 23.8 & 28.9 & 35.8 & 33.7 & 30.1 \\
\hline
\end{tabular}

353 Table 3 shows the improvement in the contingency matrix of DTs after the proposed changes; inclusion of 354 a top-level model and POI density in the DTs. The DTs determine various aspects of the activity-travel 355 pattern such as activity start time, duration, destination choice, intermediate stop type etc. It can be observed 356 that these changes and in particular POI density considerably increased the DT's explanatory power in many cases. These improvements account even above 60\%. An exception, in this case, is for DT choose Number of Work Episodes where the overall model explanatory power is reduced. However, it should be noted that the model accuracy is still above $75 \%$ in each region, therefore, these are negligible reductions.

POI density is found significant in new DTs created to specifically model location choice of primary activity. However, it is found significant in only one DT for secondary activity. The results are further elaborated in 362 Discussion (section 6).

Table 3: Improvement in Decision Trees in Activity-Based Model for medium-sized study area as compared to the Full-scale 364 model

\begin{tabular}{|l|c|c|c|}
\hline Decision Tree / Study area & Antwerp & Mechelen & Leuven \\
\hline Choose Number Of Work Episodes & $-1.49^{*}$ & $-1.55^{*}$ & $-1.27^{*}$ \\
\hline Choose Home-Based Tour Types Sequence & $5.42^{*}$ & $45.70^{*}$ & $26.65^{*}$ \\
\hline Choose HBWI1 Intermediate Stop Activities & 37.84 & 41.33 & 22.28 \\
\hline Choose HBWI2 Intermediate Stop Activities & -0.04 & 1.61 & 27.58 \\
\hline Choose HBWI12 Intermediate Stop Activities & 56.83 & 22.98 & 39.33 \\
\hline Choose HBO Intermediate Stop Types Fixed Flexible Mixed & 1.34 & $2.64^{*}$ & $-2.83^{*}$ \\
\hline Choose HBO Intermediate Stop Activities Fixed & 2.80 & -1.20 & 2.31 \\
\hline Choose HBO Intermediate Stop Activities Flexible & 1.97 & $3.05^{*}$ & $0.87^{*}$ \\
\hline Choose HBO Intermediate Stop Activities Mixed & 8.31 & $5.86^{*}$ & $16.16^{*}$ \\
\hline Choose Duration First Work Activity & -3.61 & -1.86 & -1.94 \\
\hline Choose Duration Second Work Activity & 7.31 & 4.33 & 13.49 \\
\hline Choose Duration Fixed Activities & 1.99 & $2.27^{*}$ & $0.12^{*}$ \\
\hline Choose Duration Flexible Activities & $14.79 *$ & 13.69 & 19.56 \\
\hline Choose Primary Location In Study Area Or Catchment Area & $\mathrm{x}$ & $\mathrm{x}$ & $\mathrm{x}$ \\
\hline Choose Primary Location In Home Municipality & & $\mathrm{x}$ & $\mathrm{x}$ \\
\hline Choose Primary Location In Home Subzone & $\mathrm{x}$ & $\mathrm{x}$ & $\mathrm{x}$ \\
\hline Choose Order Municipality & & & \\
\hline Choose Nearest Order Municipality & & & \\
\hline Choose Distance Band Superzone & $\mathrm{x}$ & $\mathrm{x}$ & \\
\hline Choose POI Density Superzone Catchment Area & 2.25 & 3.69 & 4.92 \\
\hline Choose Start Time Hour of Home Based Tour Primary Episode & 59.86 & 57.66 & 62.11 \\
\hline Choose Transport Mode Primary Episode & & \\
\hline + Choose Secondary Location In Study Area Or Catchment Area & & & \\
\hline It half & & & \\
\hline + Choose Secondary Location Type In Study Area 1st half & & \\
\hline
\end{tabular}




\begin{tabular}{|l|c|c|c|}
\hline+ Choose Secondary Location In Study Area 1st half & & \\
\hline+ Choose Secondary Location In Catchment Area 1st half & & & \\
\hline $\begin{array}{l}\text { Choose Start Time Hour of Home Based 1st Half Tour } \\
\text { Secondary Episode }\end{array}$ & 5.37 & $12.43^{*}$ & $3.25^{*}$ \\
\hline Choose Transport Mode Secondary Episode 1st half tour & -3.07 & 3.85 & -9.12 \\
\hline $\begin{array}{l}\text { +Choose Secondary Location In Study Area Or Catchment Area } \\
2^{\text {nd }} \text { half }\end{array}$ & $\mathrm{x}$ & & \\
\hline+ Choose Secondary Location Type In Study Area 2nd half & & & \\
\hline+ Choose Secondary Location In Study Area 2nd half & & & \\
\hline+ Choose Secondary Location In Catchment Area $2^{\text {nd }}$ half & & \\
\hline $\begin{array}{l}\text { Choose Start Time Hour of Home Based 2nd Half Tour } \\
\text { Secondary Episode }\end{array}$ & 0.16 & 1.92 & -5.63 \\
\hline Choose Transport Mode Secondary Episode 2nd half tour & -0.80 & 3.08 & -3.55 \\
\hline $\begin{array}{l}\text { Choose Start Time Hour of Home Based Tour Last Home } \\
\text { Episode }\end{array}$ & 3.11 & 4.78 & 4.58 \\
\hline $\begin{array}{l}\text { Choose Transport Mode of Home Based Tour Last Home } \\
\text { Episode }\end{array}$ & 0.27 & 2.57 & -1.40 \\
\hline
\end{tabular}

* sign shows DTs in which POI density is found to be significant, + sign indicates new DTs created to specifically model external travel, $\mathrm{x}=\mathrm{DT}$ s where POI density is found to be significant, $\mathrm{HBW}=\mathrm{Home}$ based Work, $\mathrm{HBO}=$ home based other, $\mathrm{I} 1$ = secondary activity before the primary activity, $\mathrm{I} 2$ = secondary activity after the primary activity

\section{Model Validation}

The proposed framework-changes also stresses its accurate validation in order to evaluate its effectiveness and dependability. For instance, the top-level model may result in too many or too few individuals going to the CA. Similarly, there is a possibility that the activities in CA may result in larger time spent traveling or a substantial shift in the transport mode choice. Besides, the activity pattern may be altered that may substantially affect tours. Therefore, a validation framework for an ABM should validate activities, trips as well as tours.

Therefore, this section describes the statistical validation of the results obtained. First, a validation framework is defined followed by the description of the two models used for validation and lastly the validation metrics produced.

\subsection{Validation Framework}

The validation framework presented in this study extends the framework proposed in earlier studies (Drchal et al., 2016; Petrik et al., 2018) in three dimensions: (1) expands the scope of structure to model distribution of activities between SA and CA (2) includes the tour dimension in the validation besides activities and trips and (3) disaggregate validation of the proposed measures against socioeconomic attributes of the population. In total, 11 benchmarks are proposed to comprehensively validate ABM results (Table 4Table 4). These benchmarks complement the outcome of the DTs associated with the activity pattern, start time, duration, location choice and mode choice. These benchmarks are further described according to type.

Activities: Activities are the driving force behind the Activity-based Travel Demand Models (Ben-akiva et al., 1996). Therefore, it is important to carefully validate various aspects of activities. This paper describes eleven measures for validating activity distribution across space, time and structure (Table 4). An important remark here is that there is no concept of CA in the ABM developed for Flanders model, therefore, some post-processing is required before validation Activity Distribution in CA and SA. For this, the locations 
392 outside the study area in the medium-sized model are considered as CA in the output of the full-scale model.

393 This process is repeated for each study area separately.

394 Trips: Three measures are suggested for comparing trips between a full-scale and a medium-sized ABM.

395 These include the distribution of trips performed across travel modes and also the time spent traveling.

396 Tours: Tours are also a vital aspect of ABM as these link together the two major components of ABM i.e. 397 activity and travel. Therefore, two measures are incorporated to validate the tour-consistency between 398 predicted and actual data. These measures define the number of tours and their complexity.

\section{$399 \quad 5.2$ Validation Model Description}

400 The most important step to validate model results, after defining a validation framework, is the availability 401 of a data source that is not used in the model development. In this study the model output of FEATHERS 402 for Flanders region without the CA setup have been considered for validation. For validating, the outputs of 403 the model without the CA are post-processed and the locations are labeled as inside study area or CA as in 404 the model with the CA.

\section{$405 \quad 5.3$ Aggregate Validation}

406 Table 5 shows aggregate analysis of the proposed benchmarks in Antwerp, Leuven and Mechelen region. 407 None of the benchmarks are found to be statistically different between both the models at $10 \%$ significance 408 level in Antwerp while some differences are found in other regions.

Table 4: Validation benchmarks of the Activity-Based Model

\begin{tabular}{|c|c|c|c|c|}
\hline S. No & Benchmarks & Level & Assembly & Task \\
\hline 1 & $\begin{array}{l}\text { Time spent on each } \\
\text { activity type }\end{array}$ & Activities & Time & $\begin{array}{l}\text { Distribution of time spent on each activity } \\
\text { type. Only out-of-home activities are } \\
\text { considered }\end{array}$ \\
\hline 2 & Activity start time & Activities & Time & $\begin{array}{l}\text { Distribution of activity start time in } 30 \text {-minute } \\
\text { time bins. }\end{array}$ \\
\hline 3 & $\begin{array}{l}\text { Activity Distribution } \\
\text { in CA and SA }\end{array}$ & Activities & Space & $\begin{array}{l}\text { Distribution of share of each activity-type in } \\
\text { total activities performed in CA }\end{array}$ \\
\hline 4 & $\begin{array}{l}\text { Types of activities } \\
\text { performed* }\end{array}$ & Activities & Structure & $\begin{array}{l}\text { Distribution of } \mathrm{n} \text { different activities } \\
\text { performed across m individuals. For ease, } \\
\text { only out-of-home activities are considered. }\end{array}$ \\
\hline 5 & $\begin{array}{l}\text { Number of total } \\
\text { activities }\end{array}$ & Activities & Structure & $\begin{array}{l}\text { Distribution of total activities performed } \\
\text { across individuals }\end{array}$ \\
\hline 6 & $\begin{array}{l}\text { Number of out-of- } \\
\text { home activities }\end{array}$ & Activities & Structure & $\begin{array}{l}\text { Distribution of number of out-of-home } \\
\text { activities performed across individuals }\end{array}$ \\
\hline 7 & $\begin{array}{l}\text { Number of in-home } \\
\text { activities }\end{array}$ & $\begin{array}{l}\text { Activities/ } \\
\text { Tour }\end{array}$ & Structure & $\begin{array}{l}\text { The number of times an individual returns } \\
\text { home within a simulated day. }\end{array}$ \\
\hline 8 & Tour complexity & Tour & Structure & $\begin{array}{l}\text { Distribution of share of } a \text { activities performed } \\
\text { by } m \text { individuals before returning home }\end{array}$ \\
\hline 9 & Trips by each mode & Trips & Structure & $\begin{array}{l}\text { Distribution of percentage of trips by each } \\
\text { travel mode }\end{array}$ \\
\hline 10 & $\begin{array}{l}\text { Types of transport } \\
\text { mode use }\end{array}$ & Trips & Structure & $\begin{array}{l}\text { Distribution of } i \text { transport modes used in trips } \\
\text { by } m \text { individuals }\end{array}$ \\
\hline 11 & Time spent traveling & Trips & Time & $\begin{array}{l}\text { Distribution of time spent traveling in } 10- \\
\text { minute bins }\end{array}$ \\
\hline
\end{tabular}




\begin{tabular}{|c|c|c|c|}
\hline & $\begin{array}{l}\text { Antwerp } \\
\text { Region }\end{array}$ & $\begin{array}{l}\text { Mechelen } \\
\text { Region }\end{array}$ & $\begin{array}{l}\text { Leuven } \\
\text { Region }\end{array}$ \\
\hline Criteria & P-Value & P-Value & P-Value \\
\hline Percentage of trips by each mode & 1.00 & 0.97 & 1.00 \\
\hline Types of transport mode use & 1.00 & 1.00 & 1.00 \\
\hline Time spent travelling & 0.70 & 1.00 & 0.40 \\
\hline Types of activities performed & 0.99 & 0.76 & 0.98 \\
\hline Number of in-home activities & 0.98 & 0.98 & 1.00 \\
\hline Number of out-of-home activities & 1.00 & 1.00 & 1.00 \\
\hline Number of total activities & 1.00 & 1.00 & 1.00 \\
\hline$\%$ Of time spend on each activity & 1.00 & 1.00 & 0.98 \\
\hline Tour complexity & 1.00 & 0.66 & 1.00 \\
\hline Activity start time & 1.00 & 0.87 & 0.79 \\
\hline Activity Distribution in CA and study area & 0.98 & 0.63 & 0.63 \\
\hline
\end{tabular}

414
Table 6: Classes of socioeconomic variables

\begin{tabular}{|l|c|c|c|c|c|}
\hline Group & 1 & 2 & 3 & 4 & 5 \\
\hline Age (years) & $18-34$ & $35-54$ & $55-64$ & $65-74$ & $74+$ \\
\hline Work Status & Unemployed & Employed & - & - & - \\
\hline Driving License & No & Yes & - & - & - \\
\hline Socioeconomic Class [Income $(€)]$ & $0-1249$ & $1250-2249$ & $2250-3249$ & $3250+$ & - \\
\hline Number of Cars & 0 & 1 & 2 or more & - & - \\
\hline
\end{tabular}

Some differences are found in the benchmarks in each region (Table 7-9). For instance, the distribution of Activities in $C A$ is found to be significantly different between age group four (65-74 years) and also in case of Socioeconomic Class (SEC) group one. In total, three distributions are found to be different in Mechelen and it is observed that these classes have lesser observations than average. Table 9 shows validation results for Leuven region. Time spent on activities is significantly different for age group five (75 years or above). Similarly, time spent traveling is also found to be significantly different for households having no car. This may be due to the fact that unlike most of the other measures, time spent on activities is arbitrarily grouped using 10-minute intervals. The result changes if another value is used for defining the significance level. 
Table 7: Disaggregate results of Kolmogorov-test for Antwerp region

\begin{tabular}{|c|c|c|c|c|c|c|c|c|c|c|c|c|c|c|c|c|}
\hline \multirow{2}{*}{$\begin{array}{l}\text { Criteria / Class } \\
\text { Criteria / Class } \\
\end{array}$} & \multicolumn{5}{|c|}{ Age } & \multicolumn{2}{|c|}{ Work Status } & \multicolumn{2}{|c|}{ License } & \multicolumn{4}{|c|}{ Socioeconomic Class } & \multicolumn{3}{|c|}{ Number of Cars } \\
\hline & 1 & 2 & 3 & 4 & 5 & 1 & 2 & 1 & 2 & 1 & 2 & 3 & 4 & 0 & 1 & 2 \\
\hline Activity Start Time & 1.00 & 0.79 & 0.79 & 1.00 & 0.97 & 0.79 & 0.97 & 0.79 & 0.98 & 0.97 & 0.97 & 0.97 & 0.97 & 0.97 & 0.97 & 0.53 \\
\hline Share of each transport Mode & 1.00 & 1.00 & 1.00 & 1.00 & 1.00 & 1.00 & 1.00 & 0.77 & 1.00 & 0.77 & 1.00 & 1.00 & 0.77 & 0.77 & 1.00 & 1.00 \\
\hline $\begin{array}{l}\text { Number of modes used by each } \\
\text { individual }\end{array}$ & 1.00 & 1.00 & 1.00 & 1.00 & 1.00 & 0.70 & 0.70 & 0.70 & 0.70 & 0.70 & 0.70 & 0.70 & 0.70 & 0.70 & 0.70 & 0.70 \\
\hline Time spent travelling & 0.40 & 0.99 & 0.99 & 0.76 & 0.99 & 0.99 & 0.99 & 0.99 & 0.99 & 0.99 & 0.99 & 0.99 & 0.76 & 0.99 & 0.99 & 0.76 \\
\hline Types of activities performed & 0.96 & 1.00 & 0.27 & 0.98 & 1.00 & 0.98 & 1.00 & 0.63 & 0.66 & 0.63 & 0.96 & 0.27 & 0.96 & 0.98 & 0.96 & 0.98 \\
\hline Number of In-home activities & 0.93 & 0.66 & 0.93 & 0.66 & 1.00 & 1.00 & 1.00 & 1.00 & 1.00 & 1.00 & 1.00 & 1.00 & 1.00 & 1.00 & 1.00 & 1.00 \\
\hline Number of out-of-home activities & 1.00 & 1.00 & $0.08 *$ & 0.93 & 0.93 & 1.00 & 1.00 & 1.00 & 1.00 & 1.00 & 1.00 & 1.00 & 0.93 & 1.00 & 1.00 & 0.93 \\
\hline Number of total activities & 0.93 & 0.93 & 0.93 & 0.66 & 0.93 & 1.00 & 1.00 & 0.93 & 1.00 & 1.00 & 1.00 & 1.00 & 0.93 & 1.00 & 1.00 & 0.93 \\
\hline Time spent on activities & 0.66 & 0.98 & 0.98 & 0.98 & 0.96 & 0.98 & 0.98 & 0.98 & 1.00 & 0.98 & 1.00 & 0.98 & 0.96 & 0.66 & 0.66 & 0.28 \\
\hline Tour Complexity & 0.87 & 1.00 & 1.00 & 0.82 & 1.00 & 1.00 & 1.00 & 1.00 & 1.00 & 0.82 & 0.82 & 0.87 & 1.00 & 0.87 & 1.00 & 1.00 \\
\hline Distribution of Activities in CA & 0.63 & 1.00 & 0.63 & 0.96 & 0.52 & 0.63 & 0.63 & 0.63 & 0.63 & 0.96 & 0.63 & 0.63 & 0.63 & 0.63 & 0.96 & 0.63 \\
\hline
\end{tabular}

*significantly different at $10 \%$ significance level 
Table 8: Disaggregate results of Kolmogorov-test for Mechelen region

\begin{tabular}{|c|c|c|c|c|c|c|c|c|c|c|c|c|c|c|c|c|}
\hline \multirow{2}{*}{$\frac{\text { Criteria / Class }}{\text { Criteria / Class }}$} & \multicolumn{5}{|c|}{ Age } & \multicolumn{2}{|c|}{ Work Status } & \multicolumn{2}{|c|}{ License } & \multicolumn{4}{|c|}{ Socioeconomic Class } & \multicolumn{3}{|c|}{ Number of Cars } \\
\hline & 1 & 2 & 3 & 4 & 5 & 1 & 2 & 1 & 2 & 1 & 2 & 3 & 4 & 0 & 1 & 2 \\
\hline Activity Start Time & 0.97 & 0.97 & 0.97 & 0.30 & 0.79 & 0.97 & 0.79 & 0.53 & 1.00 & 0.53 & 0.97 & 0.97 & 0.79 & 0.97 & 0.97 & 0.97 \\
\hline Share of each transport Mode & 1.00 & 1.00 & 1.00 & 1.00 & 1.00 & 1.00 & 1.00 & 1.00 & 1.00 & 1.00 & 1.00 & 1.00 & 1.00 & 1.00 & 1.00 & 1.00 \\
\hline $\begin{array}{l}\text { Number of modes used by each } \\
\text { individual }\end{array}$ & 1.00 & 1.00 & 1.00 & 0.70 & 0.70 & 1.00 & 1.00 & 1.00 & 1.00 & 1.00 & 1.00 & 1.00 & 1.00 & 1.00 & 1.00 & 1.00 \\
\hline Time spent travelling & 0.40 & 0.40 & 0.40 & 0.76 & 1.00 & 0.40 & 0.40 & 0.76 & 0.99 & 0.99 & 0.16 & 0.76 & 0.76 & 0.99 & 0.76 & 0.40 \\
\hline Types of activities performed & 0.63 & 0.98 & 0.98 & 0.63 & 0.96 & 0.66 & 0.98 & 0.96 & 0.98 & 0.96 & 0.96 & 0.98 & 0.98 & 0.96 & 0.98 & 0.98 \\
\hline Number of In-home activities & 1.00 & 1.00 & 1.00 & 1.00 & 1.00 & 1.00 & 1.00 & 1.00 & 1.00 & 1.00 & 1.00 & 1.00 & 1.00 & 1.00 & 1.00 & 1.00 \\
\hline Number of out-of-home activities & 1.00 & 0.93 & 1.00 & 0.66 & 0.93 & 1.00 & 1.00 & 1.00 & 0.93 & 1.00 & 1.00 & 0.93 & 0.93 & 0.93 & 1.00 & 0.93 \\
\hline Number of total activities & 0.93 & 0.93 & 1.00 & 0.38 & 0.18 & 1.00 & 0.93 & 0.93 & 0.93 & 1.00 & 1.00 & 0.93 & 0.93 & 0.93 & 1.00 & 0.93 \\
\hline Time spent on activities & 0.66 & 0.66 & 0.66 & $0.08 *$ & 0.27 & 0.66 & 0.28 & 0.96 & 0.28 & 0.27 & 0.66 & 0.98 & 1.00 & 0.27 & 0.66 & 0.28 \\
\hline Tour Complexity & 0.82 & 1.00 & 0.82 & 0.82 & 0.82 & 0.82 & 1.00 & 0.87 & 0.87 & 0.82 & 1.00 & 1.00 & 1.00 & 0.82 & 1.00 & 1.00 \\
\hline Distribution of Activities in CA & 0.63 & 0.96 & 0.27 & $0.02 *$ & 0.63 & 0.66 & 0.27 & 0.96 & 0.96 & $0.09 *$ & 0.27 & 0.96 & 0.96 & 0.98 & 0.96 & 0.86 \\
\hline
\end{tabular}

Table 9: Disaggregate results of Kolmogorov-test for Leuven region

\begin{tabular}{|c|c|c|c|c|c|c|c|c|c|c|c|c|c|c|c|c|}
\hline \multirow{2}{*}{$\begin{array}{l}\text { Criteria / Class } \\
\text { Criteria / Class }\end{array}$} & \multicolumn{5}{|c|}{ Age } & \multicolumn{2}{|c|}{ Work Status } & \multicolumn{2}{|c|}{ License } & \multicolumn{4}{|c|}{ Socioeconomic Class } & \multicolumn{3}{|c|}{ Number of Cars } \\
\hline & 1 & 2 & 3 & 4 & 5 & 1 & 2 & 1 & 2 & 1 & 2 & 3 & 4 & 0 & 1 & 2 \\
\hline Activity Start Time & 0.49 & 0.96 & 0.96 & 0.77 & 0.49 & 0.30 & 0.79 & 0.53 & 0.53 & $0.07 *$ & 0.79 & 0.79 & 0.97 & 0.15 & 0.79 & 0.97 \\
\hline Share of each transport Mode & 1.00 & 1.00 & 1.00 & 0.70 & 1.00 & 1.00 & 1.00 & 1.00 & 1.00 & 0.70 & 1.00 & 1.00 & 1.00 & 0.70 & 1.00 & 1.00 \\
\hline $\begin{array}{l}\text { Number of modes used by each } \\
\text { individual }\end{array}$ & 1.00 & 1.00 & 1.00 & 1.00 & 1.00 & 1.00 & 1.00 & 1.00 & 1.00 & 1.00 & 1.00 & 1.00 & 1.00 & 1.00 & 1.00 & 1.00 \\
\hline Time spent travelling & 0.76 & 0.76 & 0.16 & 0.20 & 0.40 & 0.16 & 0.40 & 0.40 & 0.40 & 0.76 & 0.40 & 0.40 & 0.99 & $\begin{array}{c}0.05 \\
*\end{array}$ & 0.16 & 0.76 \\
\hline Types of activities performed & 1.00 & 0.66 & 0.63 & 0.27 & 0.96 & 1.00 & 0.98 & 0.63 & 0.66 & 0.27 & 0.96 & 0.96 & 0.96 & 0.27 & 1.00 & 1.00 \\
\hline Number of In-home activities & 1.00 & 1.00 & 1.00 & 1.00 & 1.00 & 1.00 & 1.00 & 0.93 & 1.00 & 1.00 & 1.00 & 1.00 & 1.00 & 1.00 & 1.00 & 1.00 \\
\hline Number of out-of-home activities & 0.66 & 1.00 & 1.00 & 0.93 & 1.00 & 1.00 & 1.00 & 0.93 & 1.00 & 1.00 & 1.00 & 1.00 & 1.00 & 1.00 & 1.00 & 1.00 \\
\hline Number of total activities & 0.66 & 1.00 & 1.00 & 0.66 & 0.66 & 0.93 & 0.93 & 0.38 & 1.00 & 1.00 & 0.93 & 1.00 & 0.93 & 0.66 & 0.93 & 1.00 \\
\hline Time spent on activities & 0.66 & 0.98 & 0.98 & 0.63 & $0.09 *$ & 0.28 & 0.98 & 0.63 & 0.98 & 0.63 & 0.98 & 0.66 & 0.98 & 0.27 & 1.00 & 0.98 \\
\hline Tour Complexity & 0.33 & 0.87 & 1.00 & 0.33 & 1.00 & 1.00 & 1.00 & 1.00 & 0.87 & 0.82 & 1.00 & 0.82 & 0.87 & 0.82 & 0.87 & 1.00 \\
\hline Distribution of Activities in CA & 0.63 & 0.96 & 0.96 & $0.09 *$ & $0.09^{*}$ & 0.63 & 0.63 & 0.89 & 0.27 & 1.00 & 0.63 & 0.27 & 0.63 & 0.96 & 0.27 & 0.63 \\
\hline
\end{tabular}




\section{Discussion}

433 This paper describes a scheme to model residents external activity and travel by defining only the region of activity-travel as part of complete schedule rather than modeling them separately through external travel models. Thus, the presented methodology allows to develop an ABM for a medium-sized region by addressing the issue of external travel. Furthermore, it also reduces data collection, model development and computational efforts as the HTS and synthetic population is only required for the study area. However, defining a medium-sized region as a study area also increases non-resident external trips in the study area. therefore, proper estimation of non-residents external trips is required in order to correctly calibrate the ABM. To address this issue, a comprehensive methodology is described to estimate non-residents external trips which only rely on the open-source platforms and the HTS. For details, the readers may refer to (Baqueri, Adnan \& Bellemans, 2018; Baqueri, Adnan, Knapen, et al., 2018). Therefore, defining a mediumsized study area and properly estimating external trips is a better approach in terms of data collection and model development efforts for ABMs while estimating external trips through a non-data intensive approach.

The ABM framework proposed in this study has a generic skeleton and can be applied to any other ABM. An added value of this approach is the ability to test policy scenarios. For instance, What shall be the effect on residents' travel pattern of an improved transit service in the CA? or the effect of land use change in the CA on the distribution of activities within and outside the study area? Or implications of congestion charging around the boundary of the study area on total vehicle kilometers traveled?

There are some observations that require further explanation. For instance, the variable POI density is not found significant in the DTs that determine the location of secondary activity, except in one occasion. One reason behind this may be that the POI density is defined irrespective of the activity type that can be performed there. However, most open-source platforms allow categorizing POI according to the activity type such as work, education, shopping, etc. Thus, the POI densities can be calculated discretely for each activity type. This adaptation shall further enrich the DTs for each type of the secondary activities. Furthermore, the variation in the land use can also be effectively utilized by developing numerous indexes from the open-source data. Case in point is the Entropy Index measure which solely relies on the POI count and describes the land use as mixed or suitable only for a particular activity type (Baqueri, Adnan \& Bellemans, 2018).

Another important aspect here to consider is the quality of the open-source data. For example, the correlation between the buildup area and POI density in Antwerp, Mechelen, and Leuven is $0.68,0.67$ and 0.85 respectively. This strong association between the two data sources improved the model explanatory power and especially the top-level model. The results may be different if the two data sources do not match with each other. Therefore, a successful implementation of the proposed approach heavily depends on the quality of the open-source data. Furthermore, the POI data represents the land use just as a point and does not distinguish them on the basis of area, height, and other attributes. Therefore, a multi-story land use could be considered equivalent to a single shop. For instance, the hospital in Leuven is a super entity where patients from all over Flanders visit, thus generating a lot of external travel. However, the lack of data on its area or other characteristics undervalues its prominence. This shall be a possible explanation behind differences in some validation measures in the Leuven region.

Besides, the availability of a land use (in terms of opening hours) is also relevant for assigning locations, which many open-source platforms either do not contain at all or allow its restricted usage. However, with the advancements in the Internet of Things (IoT), further detailed information can be obtained and utilized as per the availability and the modeling requirements. Few recent studies have described the potential 


\section{References}

514 (2017).

usefulness of the open-source and the social media data for modeling travel behavior. For a comprehensive overview of the challenges and available opportunities in this regard, the readers may refer to Rashidi et al.

\section{Conclusion and Future Work}

This paper presented a framework to develop an ABM for medium-sized regions by allowing for residents' external activity-travel. Earlier studies separately modeled residents' external travel (i.e. outside the scope of the ABM) which resulted in many drawbacks such as the distortions in travel patterns as activity-locations are assigned only within the study area. Therefore, for an ABM to be effective in replicating the actual environment, an expanded study area is required to minimize the external travel.

In the proposed framework, the external locations are included in the destination choice models in the form of a CA as possible locations to perform an activity. The destination choice models are then modified with top-level models that determine the destination for each activity in the study area or CA. For activities to be performed inside the CA, a series of DTs are activated that collectively decide the destination. These DTs solely rely on individual's socioeconomic attributes, available activity-travel decisions, and open-source land use information but they do not require any detailed land use or network information as that may not be available for the CA. These modifications allow modeling external activity-travel as part of the daily travel pattern rather than estimating them through separate models which are not sensitive to policy measures. Furthermore, the proposed approach also provides an added flexibility to define the study area as per the modeling needs. These changes are implemented in ABM-FEATHERS and tested on three mediumsized regions in Flanders, Belgium. The results confirm clear advantages of the proposed methodology in terms of the decision hierarchy, model development, run-time and also data collection efforts if the ABM needs to be developed from scratch. Slight differences in validation are also found in one region where the POI density is not in a close relationship with the detailed land use data. This suggests that the availability of adequate land use information holds a central position in the proposed framework.

Furthermore, a comprehensive validation framework is also suggested to compare the model outputs obtained by defining complete Flanders as the study area and these medium-sized regions as the study areas. The validation measures include a comparison between activities, trips, and tours in terms of time, space and the structure. Furthermore, disaggregate validation is also analyzed using five socioeconomic characteristics (age, work status, driving license, income, and number of cars). The results confirm a close resemblance between both the models which suggests that an ABM can be developed for small-scale regions, once the question of external travel is addressed. This paper, therefore, shall pave the way for practitioners in developing an $\mathrm{ABM}$ for a medium-sized region.

The future work shall focus on further testing the applicability of the proposed approach. For instance, numerous policy scenarios can be tested in the study area or the CA or a case study of new transport policies/ services etc. can be studied. This way the added value of the framework can be quantified better by comparing it against a benchmark such as the full-scale ABM. This shall ultimately, therefore, lead towards developing ABM for medium-sized regions.

Adnan, M., Pereira, F.C., Azevedo, C.L., et al., (2016). Evaluating Disruption Management Strategies In Rail Transit Using SimMobility Mid-Term Simulator : A Study Of Singapore MRT North-East Line. 96th Annual Meeting, Transportation Research Board. 
Adnan, M., Pereira, F.C., Miguel, C., et al., (2016). SimMobility: A Multi-Scale Integrated Agent-based Simulation Platform. Transportation Research Board (TRB) 95th Annual Meeting, pp.1-18.

Arentze, T.A. \& Timmermans, H.J.P., (2004). A learning-based transportation oriented simulation system. Transportation Research Part B: Methodological, 38(7), pp.613-633.

Auld, J. \& Mohammadian, A.K., (2012). Activity planning processes in the Agent-based Dynamic Activity Planning and Travel Scheduling (ADAPTS) model. Transportation Research Part A: Policy and Practice, 46(8), pp.1386-1403.

Bao, Q. et al., (2016). Activity-based travel demand modeling framework feathers: Sensitivity analysis with decision trees,

Bao, Q. et al., (2015). Investigating micro-simulation error in activity-based travel demand forecasting: a case study of the FEATHERS framework. Transportation Planning and Technology, 38(4), pp.425441 .

Baqueri, S.F.A., Adnan, M., Knapen, L., et al., (2018). Modelling Distribution of External-Internal Trips and Its Intra-region and Inter-region Transferability. Arabian Journal for Science and Engineering, accepted,. Available at: http://link.springer.com/10.1007/s13369-018-3482-x.

Baqueri, S.F.A., Adnan, M. \& Bellemans, T., (2018). Modeling External Trips: Review of Past Studies and Directions for Way Forward. Journal of Transportation Engineering, Part A: Systems, 144(9). Available at: http://ascelibrary.org/doi/10.1061/JTEPBS.0000179.

Bekhor, S., Kheifits, L. \& Sorani, M., (2014). Stability analysis of activity-based models: Case study of the Tel Aviv transportation model. European Journal of Transport and Infrastructure Research, 14(4), pp.311-331.

Bellemans, T. et al., (2010). Implementation Framework and Development Trajectory of FEATHERS Activity-Based Simulation Platform. Transportation Research Record: Journal of the Transportation Research Board, 2175(1), pp.111-119.

Ben-akiva, M., Bowman, J.L. \& Gopinath, D., (1996). Travel demand model system for the information era. Transportation, 23, pp.241-266.

Bowman, J.L.. \& Ben-Akiva, M., (1998). The Day Activity Schedule Approach to Travel Demand Analysis. Transportation.

Bowman, J.L. \& Bradley, M., (2006). Activity-Based Travel Forecasting Model for SACOG,

Castiglione, J., Freedman, J. \& Bradley, M., (2003). Systematic Investigation of Variability due to Random Simulation Error in an Activity-Based Microsimulation Forecasting Model. Transportation Research Record, 1831(3), pp.76-88.

Childress, S., Nichols, B. \& Coe, S., (2015). Using an activity-based model to explore possilbe impacts of automated vehicles. Transportation Research Board 94th Annual Meeting.

Cools, M. et al., (2011). Assessment of the Effect of Micro-simulation Error on Key Travel Indices: Evidence from the Activity-Based Model FEATHERS. In Transportation Research Board 90th Annual Meeting.

Copperman, R. et al., (2016). Development of a Risk Analysis Methodology for Quantifying the Uncertainty of Travel Demand Forecasts. Available at: http://tfresource.org/images/a/a6/ITM16_Development_of_a_Risk_Analysis_Methodology_for_Qua ntifying_the_Uncertainty_of_Travel_Demand_Forecasts.pdf. 
Department for Transport, (2017). NTEM Planning Data Version 7.2,

Drchal, J., Č Ertický, M. \& Jakob, M., (2016). Data driven validation framework for multi-agent activitybased models. In Lecture Notes in Computer Science (including subseries Lecture Notes in Artificial Intelligence and Lecture Notes in Bioinformatics). pp. 55-67.

Gärling, T., Kwan, M.P. \& Golledge, R.G., (1994). Computational-process modelling of household activity scheduling. Transportation Research Part B, 28(5), pp.355-364.

Gibb, J. \& Bowman, J.L., (2007). Convergence of an activity-based travel model system to equilibrium: Experimental design and findings. In Proceedings of the 11th National Transportation Planning and Application Conference of the Transportation Research Board. Available at: http://www.trbappcon.org/2007conf/files/049 Gibb final.pdf.

Google Developers, (2017). Developers Site. Available at: https://developers.google.com/maps/documentation/business/places/ [Accessed November 3, 2016].

Hollaert, L. et al., (2012). La mobilité en Belgique en 2010 : résultats de l'enquête BELDAM,

Horckmans, M., (2017). Waalse werknemer gaat sneller taalgrens over dan Vlaamse collega. Express Business. Available at: https://nl.express.live/2017/01/26/waalse-werknemer-gaat-sneller-taalgrensdan-vlaamse-collega/ [Accessed February 27, 2018].

Hussain, I. et al., (2016). Negotiation and Coordination in Carpooling. Transportation Research Record: Journal of the Transportation Research Board, 2542, pp.92-101. Available at: http://trrjournalonline.trb.org/doi/10.3141/2542-11.

Janssens, D., Declercq, K. \& Wets, G., (2014). Onderzoek Verplaatsingsgedrag Vlaanderen 4.5 (20122013),

de Jong, G. et al., (2007). Uncertainty in traffic forecasts: Literature review and new results for The Netherlands. Transportation, 34(4), pp.375-395.

Kaddoura, I., Kröger, L. \& Nagel, K., (2017). An activity-based and dynamic approach to calculate road traffic noise damages. Transportation Research Part D: Transport and Environment, 54, pp.335347.

Knapen, L. et al., (2012). Activity-Based Modeling to Predict Spatial and Temporal Power Demand of Electric Vehicles in Flanders, Belgium. Transportation Research Record: Journal of the Transportation Research Board, 2287, pp.146-154. Available at: http://trrjournalonline.trb.org/doi/10.3141/2287-18.

Lefebvre, W. et al., (2013). Presentation and evaluation of an integrated model chain to respond to trafficand health-related policy questions. Environmental Modelling and Software, 40, pp.160-170.

Miller, E. \& Roorda, M., (2003). Prototype Model of Household Activity-Travel Scheduling. Transportation Research Record: Journal of the Transportation Research Board, 1831, pp.114-121. Available at: http://trrjournalonline.trb.org/doi/10.3141/1831-13.

OpenStreetMap contributors, (2017). https://planet.osm.org.

Petrik, O. et al., (2018). Uncertainty analysis of an activity-based microsimulation model for Singapore. Future Generation Computer Systems. Available at: http://linkinghub.elsevier.com/retrieve/pii/S0167739X1830150X.

Pieters, M., de Jong, G. \& van der Hoorn, T., (2012). Cross-border car traffic in dutch mobility Models. European Journal of Transport and Infrastructure Research, 12(2), pp.167-177. 
Prelipcean, A.C., Gidófalvi, G. \& Susilo, Y.O., (2015). Comparative framework for activity-travel diary collection systems. In 2015 International Conference on Models and Technologies for Intelligent Transportation Systems, MT-ITS 2015. pp. 251-258.

Rashidi, T.H. et al., (2017). Exploring the capacity of social media data for modelling travel behaviour: Opportunities and challenges. Transportation Research Part C: Emerging Technologies, 75, pp.197211.

Rasouli, S., (2016). Uncertainty in modeling activity-travel demand in complex urban systems. Eindhoven University of Technology.

Rasouli, S. \& Timmermans, H., (2012). Uncertainty in travel demand forecasting models: literature review and research agenda. Transportation Letters, 4(1), pp.55-73. Available at: http://www.tandfonline.com/doi/full/10.3328/TL.2012.04.01.55-73.

Recker, W.W., McNally, M.G. \& Root, G.S., (1986a). A model of complex travel behavior: Part II-An operational model. Transportation Research Part A: General, 20(4), pp.319-330. Available at: http://linkinghub.elsevier.com/retrieve/pii/0191260786900907.

Recker, W.W., McNally, M.G. \& Root, G.S., (1986b). A model of complex travel behavior: Part II-An operational model. Transportation Research Part A: General, 20(4), pp.319-330. Available at: http://linkinghub.elsevier.com/retrieve/pii/0191260786900907.

Shabanpour, R. et al., (2016). Investigating the applicability of ADAPTS activity-based model in air quality analysis. Travel Behaviour and Society.

Shiftan, Y., Kheifits, L. \& Sorani, M., (2015). Travel and Emissions Analysis of Sustainable Transportation Policies with Activity-Based Modeling. Transportation Research Record: Journal of the Transportation Research Board, 2531, pp.93-102. Available at: http://trrjournalonline.trb.org/doi/10.3141/2531-11.

Siyu, L.I., (2015). Activity-Based Travel Demand Model : Application And Innovation. Department of Civil and Environmental Engineering National University of Singapore.

Smith, L., Beckman, R. \& Baggerly, K., (1995). TRANSIMS: Transportation analysis and simulation system. Other Information: PBD: [1995], (26295). Available at:

http://www.osti.gov/scitech//servlets/purl/88648-fgWOUT/webviewable/.

Statbel, (2017). Land use in Belgium since 1990. Available at: https://statbel.fgov.be/nl/themas/leefmilieu/grond/bodemgebruik\#figures.

Usman, M. et al., (2017). Optimal recharging framework and simulation for electric vehicle fleet. Future Generation Computer Systems. Available at: http://linkinghub.elsevier.com/retrieve/pii/S0167739X17307689 [Accessed July 27, 2017].

Veldhuisen, J., Timmermans, H. \& Kapoen, L., (2000). Microsimulation Model of Activity-Travel Patterns and Traffic Flows: Specification, Validation Tests, and Monte Carlo Error. Transportation Research Record: Journal of the Transportation Research Board, 1706, pp.126-135. Available at: http://trrjournalonline.trb.org/doi/10.3141/1706-15.

Weiss, C. et al., (2017). Assessing the effects of a growing electric vehicle fleet using a microscopic travel demand model. European Journal of Transport and Infrastructure Research, 17(3).

Zhuge, C. et al., (2017). Sensitivity analysis of integrated activity-based model: using MATSim as an example. Transportation Letters, pp.1-11. Available at: https://www.tandfonline.com/doi/full/10.1080/19427867.2017.1286772. 